\title{
Confirmation via the Continuum-Fitting Method That the Spin of the Black Hole in Cygnus X-1 Is Extreme
}

\section{Citation}

Gou, Lijun, Jeffrey E. McClintock, Ronald A. Remillard, James F. Steiner, Mark J. Reid, Jerome A. Orosz, Ramesh Narayan, Manfred Hanke, and Javier García. 2014. "Confirmation via the Continuum-Fitting Method That the Spin of the Black Hole in Cygnus X-1 Is Extreme." The Astrophysical Journal 790 (1) (June 30): 29. doi:10.1088/0004-637x/790/1/29. http:// dx.doi.org/10.1088/0004-637X/790/1/29.

\section{Published Version}

doi:10.1088/0004-637X/790/1/29

\section{Permanent link}

http://nrs.harvard.edu/urn-3:HUL.InstRepos:27802019

\section{Terms of Use}

This article was downloaded from Harvard University's DASH repository, and is made available under the terms and conditions applicable to Other Posted Material, as set forth at http:// nrs.harvard.edu/urn-3:HUL.InstRepos:dash.current.terms-of-use\#LAA

\section{Share Your Story}

The Harvard community has made this article openly available. Please share how this access benefits you. Submit a story. 


\title{
CONFIRMATION VIA THE CONTINUUM-FITTING METHOD THAT THE SPIN OF THE BLACK HOLE IN CYGNUS X-1 IS EXTREME
}

\author{
Lijun Gou ${ }^{1,2}$, Jeffrey E. McClintock ${ }^{2}$, Ronald A. Remillard ${ }^{3}$, James F. Steiner ${ }^{2}$, Mark J. Reid ${ }^{2}$, Jerome A. Orosz ${ }^{4}$, \\ RaMesh NARAYAN ${ }^{2}$, MANFred HankE ${ }^{5}$, AND JAVIER García ${ }^{2}$ \\ ${ }^{1}$ National Astronomical Observatory, Chinese Academy of Science, Beijing 100012, China \\ ${ }^{2}$ Harvard-Smithsonian Center for Astrophysics, Cambridge, MA 02138, USA \\ ${ }^{3}$ Kavli Institute for Astrophysics and Space Research, MIT, 70 Vassar Street, Cambridge, MA 02139, USA \\ ${ }^{4}$ Department of Astronomy, San Diego State University, 5500 Campanile Drive, San Diego, CA 92182, USA \\ ${ }^{5}$ Remeis-Observatory \& ECAP, University of Erlangen-Nuremberg, Sternwartstrasse 7, D-96049 Bamberg, Germany \\ Received 2013 August 14; accepted 2014 June 4; published 2014 June 30
}

\begin{abstract}
In Gou et al., we reported that the black hole primary in the X-ray binary Cygnus X-1 is a near-extreme Kerr black hole with a spin parameter $a_{*}>0.95(3 \sigma)$. We confirm this result while setting a new and more stringent limit: $a_{*}>0.983$ at the $3 \sigma(99.7 \%)$ confidence level. The earlier work, which was based on an analysis of all three useful spectra that were then available, was possibly biased by the presence in these spectra of a relatively strong Compton power-law component: the fraction of the thermal seed photons scattered into the power law was $f_{\mathrm{s}}=23 \%-31 \%$, while the upper limit for reliable application of the continuum-fitting method is $f_{\mathrm{s}} \lesssim 25 \%$. We have subsequently obtained six additional spectra of Cygnus X-1 suitable for the measurement of spin. Five of these spectra are of high quality with $f_{\mathrm{s}}$ in the range $10 \%-19 \%$, a regime where the continuum-fitting method has been shown to deliver reliable results. Individually, the six spectra give lower limits on the spin parameter that range from $a_{*}>0.95$ to $a_{*}>0.98$, allowing us to conservatively conclude that the spin of the black hole is $a_{*}>0.983(3 \sigma)$.
\end{abstract}

Key words: black hole physics - X-rays: binaries - X-rays: individual (Cygnus X-1)

Online-only material: color figures

\section{INTRODUCTION}

The X-ray binary Cygnus X-1 was discovered in the early days of X-ray astronomy (Bowyer et al. 1965), and its compact primary was the first black hole candidate to be established via dynamical observations (Webster \& Murdin 1972; Bolton 1972). Recently, in three sequential papers on Cygnus X-1, we reported accurate values of the source distance $D$ (Reid et al. 2011), black hole mass $M$, and orbital inclination angle $i$ (Orosz et al. 2011), and an extreme value for the black hole's spin parameter, $a_{*}>0.95\left(3 \sigma\right.$; Gou et al. 2011). ${ }^{6}$

We measured the spin of the black hole by fitting the thermal $\mathrm{X}$-ray continuum spectrum of the accretion disk to the thin-disk model of Novikov \& Thorne (1973). The key fit parameter is the radius of the inner edge of the accretion disk, which is equivalent to the radius of the innermost stable circular orbit $R_{\mathrm{ISCO}}$ (Zhang et al. 1997; McClintock et al. 2013). In turn, $R_{\mathrm{ISCO}} / M$ is directly related to the dimensionless spin parameter $a_{*}$ (Bardeen et al. 1972). The continuum-fitting method of measuring spin is simple: It is strictly analogous to measuring the radius of a star whose flux, temperature, and distance are known. By this analogy, it is clear that one must have accurate estimates of $D, M$, and $i$ in order to obtain an accurate estimate of $a_{*}$ by fitting the X-ray spectrum. The robustness of the continuumfitting method is demonstrated by the dozens or hundreds of independent and consistent measurement of spin that have been obtained for several black holes (e.g., Steiner et al. 2010), and through careful consideration of many sources of systematic errors (e.g., Steiner et al. 2011; Kulkarni et al. 2011; Zhu et al. 2012).

6 The dimensionless spin parameter $a_{*} \equiv c J / G M^{2}$ with $\left|a_{*}\right| \leqslant 1$, where $J$ is the angular momentum of the black hole.
Herein, using the continuum-fitting method (McClintock et al. 2013) and precisely the same methodologies that are described in Gou et al. (2011; hereafter GOU11) — but using data of much higher quality - we confirm our conclusion that Cygnus X-1's black hole is a near-extreme Kerr hole, a result that has received support via the independent Fe-line method of measuring spin (see Section 7.1). Importantly, these new data allow us to obtain a more stringent limit on the spin parameter, namely, $a_{*}>0.983(3 \sigma)$.

For reliable application of the continuum-fitting method, it is essential that the thermal disk component dominate over the Compton power-law component (McClintock et al. 2013), which is always present in the spectra of X-ray binaries. It is by this criterion that the present data are of much higher quality than those analyzed in GOU11, as we now explain. The strength of the complicating Compton component is parameterized by the scattering fraction $f_{\mathrm{s}}$, which is the fraction of the thermal seed photons that are scattered into the power-law component (Steiner et al. 2009b). Ideally, $f_{\mathrm{s}}$ is a few percent, while the limit for reliable application of the continuum-fitting method, based on a thorough investigation of two black hole binaries, has been shown to be $f_{\mathrm{s}} \lesssim 25 \%$ (Steiner et al. 2009a). The extreme spin reported in GOU11 is based on an analysis of the only three spectra of Cygnus X-1 that were then available and suitable for measurement of spin via the continuum-fitting method. One spectrum was marginally within the limit $\left(f_{\mathrm{s}}=23 \%\right)$ and the other two were above the limit (both with $f_{\mathrm{s}}=31 \%$ ). Herein, we report on spin results for six new spectra, five of which have much more favorable scattering fractions in the range $f_{\mathrm{s}}=10 \%-19 \%$. Each of the six spectra individually confirms the spin limit set by GOU11 $\left(a_{*}>0.95\right.$ at $\left.3 \sigma\right)$.

It is challenging to measure the spin of Cygnus X-1 not only because the Compton component is always relatively strong for 


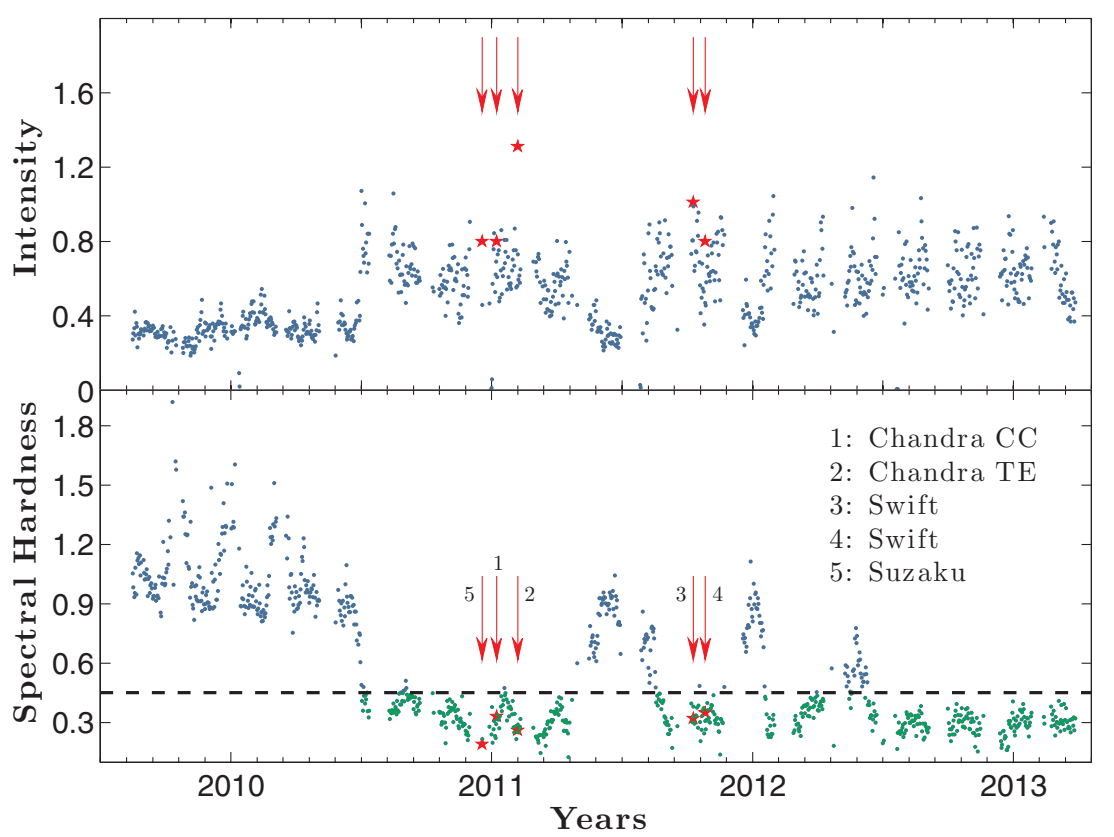

Figure 1. Intensity of Cygnus $X-1$ in the $2-10 \mathrm{keV}$ band relative to the Crab Nebula and its pulsar, and spectral hardness (bottom) based on data obtained using the MAXI Gas Slit Camera (GSC; Mihara et al. 2011). The spectral hardness (SH) is defined as the ratio of counts detected in a hard X-ray band (4-10 keV) to those detected in a soft band $(2-4 \mathrm{keV})$. As an empirical choice, we measure spin using only those data for which the spectral hardness is below the dashed line (SH $<$ 0.45). Shown plotted as red stars are the intensity and hardness of the source as observed by MAXI on the days of the five observations listed in Table 1. The survey data are useful for the purposes of data selection, but they are unsuitable for the measurement of spin.

(A color version of this figure is available in the online journal.)

this source (e.g., see Section 4.3 in McClintock \& Remillard 2006), but also for two additional reasons: (1) it is essential to have spectral data that span a broad energy range, $\sim 0.5-40 \mathrm{keV}$, in order to simultaneously constrain the unusually soft thermal component $(k T \sim 0.5 \mathrm{keV})$ and the Compton power-law and reflected components (see Section 2 and Figure 3 in GOU11), and such broadband data are rare; and (2) the source dwells in its soft state only a small fraction of the time. ${ }^{7}$ In mid-2010, Cygnus X-1 again entered the soft state. Seizing this opportunity, we observed the source with Chandra, Swift, Suzaku, and RXTE and obtained the spectra with moderate values of $f_{\mathrm{s}}$ that are mentioned above. The times of these various observations are indicated by arrows in the X-ray light curve shown in Figure 1.

The paper is organized as follows. In Section 2, we describe the observations and data reduction, and in Section 3 the data analysis and our spectral model. Presented in Sections 4, 5, and 6 , respectively, are our results, a discussion of their robustness, and a comprehensive analysis of the errors. In Section 7, we first discuss spin results obtained using the Fe-line method and then compare Cygnus X-1 to two other well-studied persistent black hole systems. We offer our conclusions in the final section.

\section{OBSERVATIONS AND DATA REDUCTION}

In late 2010 and during 2011, we made the five soft-state observations listed in Table 1 using Chandra, Swift, Suzaku, and RXTE. For the four Chandra and Swift observations, the essential high-energy coverage was provided via simultaneous observations made using the Proportional Counter Array (PCA) aboard RXTE. Because RXTE observations are segmented by Earth occultations and because we require that the RXTE observations be strictly simultaneous (with those of Chandra

\footnotetext{
7 Fourteen years of continuous monitoring data show that the source spectrum was suitably soft only about $10 \%$ of the time (see Figure 1 in GOU11, with attention to those data in the lower panel that fall below the dashed line).
}

or Swift), we chopped the 5 observations into 10 observation intervals, each providing 1 of the spectra S1-S10 that are listed in Table 1. Here and throughout, "spectrum" refers to a segment of an observation, as schematically defined in Table 1. While two spectra may be part of a single contiguous observation, any two observations were obtained at disjoint time intervals and correspond to distinct pointings. We adhere to this language throughout.

Observation No. 1, which corresponds to spectra S1-S5 (Table 1), is by far the most important observation because the Compton component is relatively faint, much fainter than during Observations 2-5, and also much fainter than during the three observations reported on in GOU11. For this crucial observation, we show in Figure 2 the count rates measured by RXTE in $16 \mathrm{~s}$ bins and Chandra in $100 \mathrm{~s}$ bins.

We now discuss in turn the observations and data reduction procedures for Chandra and Swift, and then for RXTE, which provides the complementary high-energy coverage. In the final subsection, we discuss Observation No. 5, which was performed solely by $S u z a k u$, with the high-energy coverage provided by Suzaku's Hard X-ray Detector (HXD). Table 1 gives for each observation basic information including the energy range used in analyzing the data for a given detector, the gross observation times, the effective exposure times, the intensity of the source in Crab units, the spectral hardness (Figure 1), and the orbital phase of the binary system. The orbital phase is useful for assessing the likelihood that an observation is affected by absorption dips, which are observed in both the hard and soft states of Cygnus X-1 near phase zero (e.g., Hanke et al. 2009; Yamada et al. 2013).

\subsection{Observation No. 1: Chandra-Continuous Clocking (CC)}

This key observation $(\mathrm{ObsID}=12472)$ was obtained in the ACIS CC mode. As indicated in Figure 2 and discussed above, the observation, which has a total duration of $24 \mathrm{ks}$, was parceled up into five data segments. The start and stop times for each 
Table 1

Journal of the Observations ${ }^{\text {a }}$

\begin{tabular}{|c|c|c|c|c|c|c|c|c|c|}
\hline $\begin{array}{l}\text { Obs. } \\
\text { No. }\end{array}$ & $\begin{array}{l}\text { Spec. } \\
\text { No. }\end{array}$ & Mission & Detector & $\begin{array}{c}E 1-E 2 \\
(\mathrm{keV})\end{array}$ & UT & $\begin{array}{l}T_{\exp } \\
(\mathrm{s})\end{array}$ & $\begin{array}{c}I \\
(\mathrm{Crab})\end{array}$ & $\mathrm{SH}$ & $\phi$ \\
\hline 1 & S1 & Chandra and RXTE & HETG(CC) and PCA & $0.8-8.0$ and $2.9-50$ & 2011 Jan 6 14:06:40-14:35:44 & 455 and 1744 & 0.52 & 0.24 & 0.32 \\
\hline 1 & $\mathrm{~S} 2$ & Chandra and $R X T E$ & HETG(CC) and PCA & $0.8-8.0$ and $2.9-50$ & 2011 Jan 6 15:44:16-16:09:52 & 398 and 1536 & 0.61 & 0.44 & 0.33 \\
\hline 1 & S3 & Chandra and $R X T E$ & HETG(CC) and PCA & $0.8-8.0$ and $2.9-50$ & 2011 Jan 6 17:15:28-17:43:44 & 462 and 1696 & 0.57 & 0.33 & 0.35 \\
\hline 1 & S4 & Chandra and $R X T E$ & HETG(CC) and PCA & $0.8-8.0$ and $2.9-50$ & 2011 Jan 6 18:19:44-19:17:52 & 997 and 3488 & 0.38 & 0.26 & 0.36 \\
\hline 1 & S5 & Chandra and $R X T E$ & HETG(CC) and PCA & $0.8-8.0$ and $2.9-50$ & 2011 Jan 6 19:53:36-20:50:08 & 847 and 3392 & 0.38 & 0.22 & 0.37 \\
\hline 2 & S6 & Chandra and $R X T E$ & HETG(TE) and PCA & $0.5-10.0$ and $2.9-50$ & 2011 Feb 5 07:02:00-09:37:00 & 3593 and 3600 & 0.58 & 0.25 & 0.64 \\
\hline 2 & S7 & Chandra and $R X T E$ & HETG(TE) and PCA & $0.5-10.0$ and $2.9-50$ & 2011 Feb 5 10:10:00-10:31:00 & 929 and 1232 & 0.74 & 0.31 & 0.65 \\
\hline 3 & S8 & Swift and $R X T E$ & XRT(WT) and PCA & $0.5-10.0$ and $2.9-50$ & 2011 Oct 8 20:03:28-20:26:08 & 1355 and 1344 & 0.59 & 0.32 & 0.48 \\
\hline 3 & S9 & Swift and $R X T E$ & $\mathrm{XRT}(\mathrm{WT})$ and PCA & $0.5-10.0$ and $2.9-50$ & 2011 Oct 8 21:40:00-22:02:08 & 1326 and 1328 & 0.90 & 0.28 & 0.49 \\
\hline 4 & $\mathrm{~S} 10$ & Swift and RXTE & XRT(WT) and PCA & $0.5-10.0$ and $2.9-50$ & 2011 Oct 26 03:28:00-04:10:00 & 1454 and 2464 & 0.47 & 0.35 & 0.57 \\
\hline 5 & $\mathrm{~S} 11$ & Suzaku & XIS and HXD & $0.5-10.0$ and $2.5-45$ & 2010 Dec 17 14:31:07-18:49:22 & 868 & & 0.19 & 0.77 \\
\hline
\end{tabular}

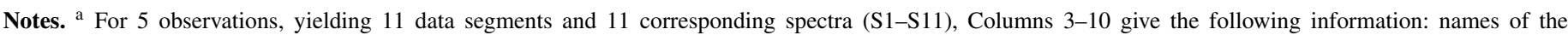

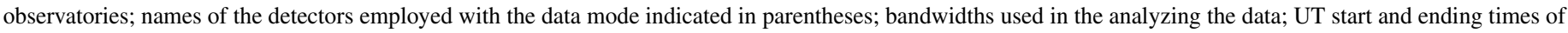

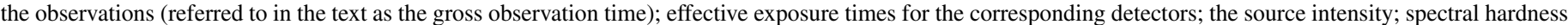

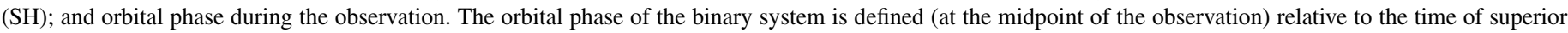
conjunction of the O-star (black hole beyond star), which occurred on heliocentric Julian Day 2441163.529 (Orosz et al. 2011).

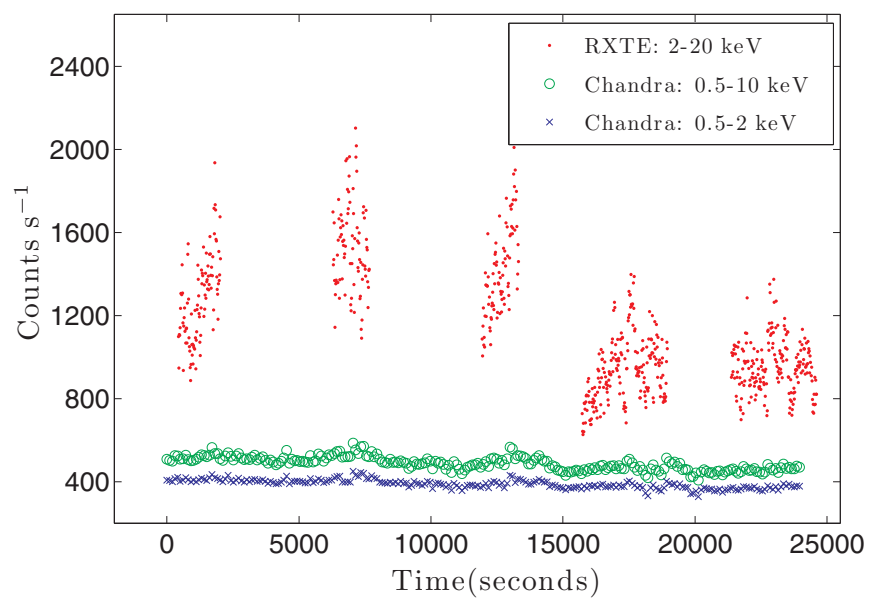

Figure 2. RXTE and Chandra count rates in the energy bands indicated for Observation No. 1. The strictly simultaneous segments of data used to produce the five spectra of highest quality, namely, S1-S5, correspond in the figure to the five time intervals defined by the five clusters of $R X T E$ data points (red filled circles). The UT start and stop times of each of these five time intervals are given in Table 1 . Note the strong variability in the RXTE band, where the Compton component dominates, relative to the Chandra bands, where the thermal component dominates.

(A color version of this figure is available in the online journal.)

data segment, which are given in Table 1, are the same as those for the corresponding RXTE PCA observation (Figure 2). The individual PCA observation times range from $1.5 \mathrm{ks}$ to $3.4 \mathrm{ks}$, while the corresponding Chandra net exposure times are $\approx 4$ times shorter (Table 1) due to the telemetry saturation.

For this Chandra observation, as well as for Observation No. 2 (see Section 2.2), we (1) used the High-Energy Transmission Grating (HETG) and the Advanced Camera for Imaging and Spectroscopy (ACIS-S; Garmire et al. 2003; Canizares et al. 2005); (2) binned the data to achieve a minimum number of counts per channel of $200 ;{ }^{8}$ and (3) made no allowance

\footnotetext{
8 The bin size used is approximately two to four times larger than the default grating resolution, as recommended for modeling the continuum http://cxc.harvard.edu/ciao/threads/spectra_grouping/. The fit results are unchanged if the data are binned more finely, although reduced chi-squared will be slightly lower.
}

for systematic error because the statistical error is completely dominant for all our Chandra data; e.g., adding in each channel a systematic error of $1 \%$ in quadrature with the statistical error leaves our fit results unchanged.

Observing a bright source such as Cygnus X-1 is challenging because of the effects of "pileup," i.e., the arrival of two or more photons in the same or adjacent pixel within a single frame time. The $\mathrm{CC}$ mode has the virtue of a short $2.85 \mathrm{~ms}$ frame time that is achieved by continuously transferring the data from the image array to the frame-store array. While this largely solves the problem of pileup (see Section 5.2.1), it results in the collapse of the two-dimensional image into a onedimensional image and a consequent loss of information on the spatial distribution of photons (also see Section 2 in GOU11). Telemetry limitations are also a consideration in observing a bright source. Accordingly, we only transmitted the data for the high-energy grating (HEG; -1 order) and medium-energy grating (MEG; +1 order) components of the HETG. We used the standard procedures for extracting the data, ${ }^{9}$ which (apart from the 1.3-2.0 keV chip gap in the MEG spectrum) were fitted over the energy range $0.8-8.0 \mathrm{keV}$.

\subsection{Observation No. 2: Chandra-Timed Exposure (TE)}

In reducing these TE-mode data $(\mathrm{ObsID}=13219)$, we followed the method described by Smith et al. (2002) while again using the orders of the HEG and MEG spectra mentioned above. For the TE-mode data we also used the readout "streak" spectra located alongside the HEG and MEG spectra. We followed the recommended procedures in extracting the streak and background spectra. ${ }^{10}$ Although the net exposure times for the two TE-mode spectra S6 and S7 are, respectively, $3.6 \mathrm{ks}$ and $0.9 \mathrm{ks}$, the effective exposure times for the streak spectra are only about $19.2 \mathrm{~s}$ and $5.0 \mathrm{~s}$, respectively. As in GOU11, we used the full $0.5-10 \mathrm{keV}$ bandwidth for the streak spectra, which has a pileup fraction that is less than $3 \%$ over the whole range. For the HEG and MEG spectra, we used the energy ranges $0.7-0.9 \mathrm{keV}$ and 7.0-10.0 keV and confirmed that the pileup fraction in these

\footnotetext{
9 http://cxc.harvard.edu/ciao/threads/spectra_hetgacis/

$10 \mathrm{http} / / / \mathrm{cxc}$. harvard.edu/ciao/threads/streakextract/
} 
energy intervals is less than 5\%. (See Section 5.2 for a discussion of pileup effects.)

\subsection{Observation Nos. 3-4: Swift-Windowed Timing (WT)}

Three Swift/RXTE observations were performed on UT October 8, 24, and 26. We disregard the observation of October 24 because the RXTE data were not simultaneous and the source was highly variable during this period. The WT mode was used to minimize the effects of pileup. The data were extracted using the procedures outlined in Romano et al. (2006). ${ }^{11}$ We used for the background region an extraction aperture of $50 \times 20$ pixels and for the source region $40 \times 20$ pixels (i.e., 40 pixels along the image strip and 20 pixels transverse to it; 1 pixel $=2.36$ arcsec). Despite our use of the WT mode, the data are strongly affected by pileup. Pileup is negligible below 100 counts $\mathrm{s}^{-1}$ and moderate below 300 counts s $^{-1}$ (Romano et al. 2006). However, the count rate exceeded 800 counts $\mathrm{s}^{-1}$ for all of our observations. We therefore excluded a $15 \times 20$ pixel region in the center of the source extraction region to ensure that pileup effects are small (see Section 5.2.3 for details).

We netted three simultaneous Swift $+R X T E$ observations, each $>1 \mathrm{ks}$ in duration (Table 1), that we used to measure spin. Although the gap between the two observations is only $\approx 30$ minutes, we chose not to combine them because our model fits show strong source variability, with the source intensity increasing from $0.59 \mathrm{Crab}$ to $0.90 \mathrm{Crab}$ (Table 1) and the scattering fraction increasing from $31 \%$ to $50 \%$ (Section 3 ). We binned all the Swift data to achieve a minimum of 200 counts per channel, and we included a systematic error of $0.5 \%$ in the count rates in each pulse height amplitude channel.

\subsection{Observation Nos. 1-4: RXTE}

As in GOU11, for RXTE we used only the data for PCU2, which is widely regarded as the best-calibrated detector. Meanwhile, it is unimportant whether one uses PCU2 alone or all the PCUs (GOU11). All the RXTE spectra have been reprocessed using the latest PCA calibrations available in NASA software release HEAsoft 6.13. In particular, we generated new response files and used the latest assignments for converting pulse-height channel to energy. In addition, we used a revised PCA background model, "pca_bkgd_cmvle_eMv20111129.mdl," which we obtained from the PCA instrument team. Furthermore, we corrected the effective area of the PCA using the Toor \& Seward (1974) spectrum of the Crab Nebula precisely as described in Section 2 in GOU11, thereby obtaining for Observation Nos. 1/2/3/4 normalization correction factors $C_{\mathrm{TS}}$ of $1.128 / 1.133 / 1.123 / 1.123$ and power-law slope correction factors $\Delta \Gamma_{\mathrm{TS}} 0.022 / 0.024 / 0.023 / 0.023$; the respective dead time correction factors are 1.029/1.039/1.048/1.048. Finally, as customary for PCA observations of bright sources, we included an allowance of $0.5 \%$ for systematic error. We fitted the $R X T E$ spectra over the energy range 2.9-50 keV (pulse-height channels 4-83).

\subsection{Observation No. 5: Suzaku}

Both the X-ray Imaging Spectrometer (XIS) and the HXD were used for this observation with a gross observing time of $\approx 5 \mathrm{ks}$ (Table 1 ). We reduced the data using the standard procedures described in Yamada et al. (2012). There is no fast readout mode for the XIS detector, and the effects of pileup are

11 See also http://www.swift.ac.uk/analysis/xrt/pileup.php. large, even though we excluded the counts in the central source region within a radius of 60 pixels. To achieve an acceptable fit $\left(\chi^{2}<2.0\right)$, for the XIS we ignored the energy ranges: 1.7-1.9 keV and 2.1-2.3 keV, and for the HXD we ignored the energy range below $20 \mathrm{keV}$. We furthermore added the $2 \%$ customary systematic error for the XIS. (No systematic error was included for the HXD.) Given (1) that the fit we were able to achieve is relatively poor with $\chi_{v}^{2}=1.69$, (2) the lack of any constraint on the reflection component in the 10-20 keV band, and (3) the significant effects of pileup, we do not use the Suzaku spectrum to estimate spin, although for completeness we list the observation in Table 1.

\section{DATA ANALYSIS}

A soft-state spectrum of Cygnus X-1 consists of thermal, power-law, and reflected components, which are illustrated in Figure 3. The latter component includes the $\mathrm{Fe} \mathrm{K} \alpha$ emission line. A schematic sketch of the physical structures that generate the three spectral components, namely, the accretion disk and the corona, are shown in Figure 2 in GOU11.

The spectral fitting package XSPEC ${ }^{12}$ version 12.6.0 (Arnaud 1996) was used for all data analysis and model fitting. Unless otherwise indicated, the error on a single parameter is reported at the $1 \sigma(68.3 \%)$ level of confidence. In this section and the one that follows, the input parameters $D, i$, and $M$ are fixed at their fiducial values (see Section 4).

In GOU11, we analyzed three spectra of Cygnus X-1 using a progression of seven preliminary models. The first three models, NR1-NR3, were nonrelativistic, with the accretion disk component modeled using DISKBB. The results for the physically most realistic of these models, NR3, were adopted. We obtained consistent values of inner-disk temperature and radius for the three spectra, which are reported in Table 7 in GOU11: $T=0.538 \pm 0.006$, and $R_{\text {in }}=2.12 \pm 0.15 G M / c^{2}$ (std. dev.; $N=3$ ).

Next, we analyzed the spectra using four preliminary relativistic models, R1-R4. The principal component of these models is KERRBB2 (McClintock et al. 2013), which is a fully relativistic model of a thin accretion disk. Like DISKBB, KERRBB2 returns two fit parameters, $a_{*}$ and the mass accretion rate $\dot{M}$ (instead of $T_{\text {in }}$ and $R_{\text {in }}$ ). The models R1-R4 progress toward our adopted model, where R1 is the most primitive model. The four models and our adopted model all gave very similar results for the key parameter $a_{*}$. In GOU11, we presented a full set of results for models R1-R4 to show clearly that our results for the spin parameter are insensitive to the analysis details, as expected given the dominance of the thermal component.

In this paper, we employ a single model, namely, the one adopted in GOU11, which is the most physically realistic of the eight models considered by GOU11. The structure of the model, showing all the components of which it is comprised, is expressed as follows:

\section{$\mathrm{CRABCOR} * \mathrm{CONST} * \mathrm{TBABS}[\mathrm{SIMPLR} \otimes \mathrm{KERRBB} 2$ + KERRDISK + KERRCONV $\otimes($ IREFLECT $\otimes$ SIMPLC) $]$}

To review, the power-law component is generated by SIMPLR operating on the thermal seed photons supplied by KERRBB2, while the reflection component is generated in turn by IREFLECT operating on the power-law component. The fit returns a single value of $a_{*}$, a key parameter that appears in KERRBB2, KERRDISK,

\footnotetext{
$12 \mathrm{XSPEC}$ is available at http://heasarc.gsfc.nasa.gov/xanadu/xspec/.
} 


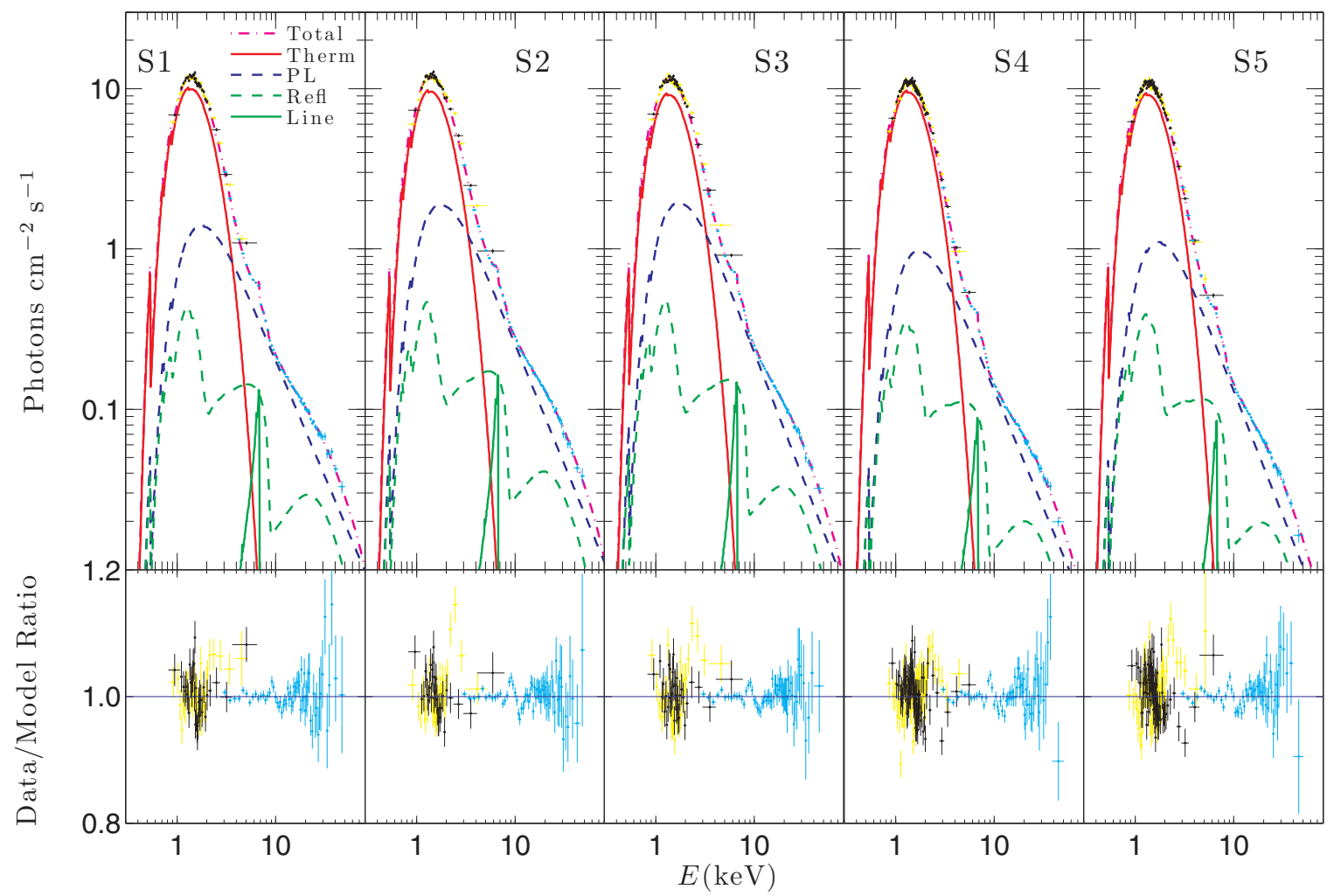

Figure 3. Top: in each spectrum, the upper envelope shows the best-fit model and the data (Chandra in black and RXTE in blue). Also shown are the thermal, power-law, and reflection components; the latter component consists of the F K $\alpha$ line plus a continuum component. (The color assignments are the same as those used in Figure 2 in GOU11.) The effect of the low-energy absorption component is apparent at energies $\lesssim 1 \mathrm{keV}$. In all of the spectra, note the dominance of the key thermal component. Bottom: a ratio plot showing the deviations between the model and the data.

(A color version of this figure is available in the online journal.)

and KERRCONV. We now discuss the principal components of the model (thermal, power-law, and reflected) and their relationships. (For further details and a complete description of each component, see GOU11.)

Thermal component. The core component is the fully relativistic thin-disk model KERRBB2 (Li et al. 2005; McClintock et al. 2013). The effects of spectral hardening are incorporated into the basic model KERRBB via a pair of look-up tables for the hardening factor $f$ corresponding to two representative values of the viscosity parameter: $\alpha=0.01$ and 0.1 (for details, see McClintock et al. 2013). Throughout this work, we use $\alpha=0.1$ unless stated otherwise (King et al. 2007). As noted earlier, the two fit parameters of KERRBB2 are $a_{*}$ and $\dot{M}$, which along with $M$ determine the Eddington-scaled bolometric luminosity of the disk, $L\left(a_{*}, \dot{M}\right) / L_{\text {Edd }}$. As in GOU11, we turn on the effects of returning radiation and limb darkening, set the torque at the inner disk radius to zero, fix the normalization to unity, allow $\dot{M}$ to vary freely, and fit directly for $a_{*}$.

Power-law component. The term SIMPLR $\otimes$ KERRBB 2 models the power-law plus the observed thermal component. SIMPLR (Steiner et al. 2011) has the same two parameters as its parent model SIMPL (Steiner et al. 2009b): the power-law photon index $\Gamma$ and the scattered fraction $f_{\mathrm{s}}$. However, SIMPLR has one additional parameter, namely, the fraction of the power-law photons that strike the disk. In this application, SIMPLR models a corona that scatters half the thermal seed photons outward and the remainder downward toward the disk, thereby generating the reflected component.

Reflected component. The remaining two additive terms inside the square brackets model the reflected component. SIMPLC, which is the isolated Compton component that illuminates the disk, is equivalent to SIMPLR $\otimes$ KERRBB 2 minus the unscattered portion of the thermal component (Steiner et al. 2011). The reflected spectrum generated by IREFLECT acting on SIMPLC contains numerous sharp absorption features but no emission lines. We supplement this partial reflection model by employing the line model KERRDISK and the convolution smearing model KERRCONV (Brenneman \& Reynolds 2006). ${ }^{13}$ We model the emissivity profile as a single power law with index $q$, and tie together all the common parameters of these two models, including the two principal parameters $a_{*}$ and $q$. (For further details concerning assumed values of elemental abundances, disk temperature, etc., see GOU11).

The three multiplicative model components are (1) CRABCOR, which corrects for calibration deviations relative to Toor \& Seward (see Section 2 in GOU11 and Steiner et al. 2011); (2) CONST, which allows for discrepancies in the calibrations of the various detectors (the normalization of the RXTE/PCU2 detector is fixed to unity and the normalizations of the Chandra and Swift detectors are allowed to float); and (3) TBABS a standard low-energy absorption model (Wilms et al. 2000).

Comparing Figure 3 with the corresponding Figure 3 in GOU11, one sees at a glance that spectra S1-S5 (with $f_{\mathrm{s}}=$ $10 \%-19 \%$ ) are much more strongly disk-dominated than spectra SP1-SP3 in GOU11 (with $f_{\mathrm{s}}=23 \%-31 \%$ ). For spectra S1-S5, the peak flux in the thermal component is 5-10 times the peak flux in the power-law component, and it is $\approx 25$ times the peak flux in the reflected component.

\footnotetext{
13 Our results are essentially unchanged if we instead use RELLINE and RELCONV (Dauser et al. 2010).
} 
Table 2

Fit Results for Observation No. 1: Spectra S1-S5

\begin{tabular}{|c|c|c|c|c|c|c|c|}
\hline Number & Model & Parameter & S1 & S2 & S3 & S4 & S5 \\
\hline 1 & KERRBB2 & $a_{*}$ & $0.99990_{-0.00877}^{+0.00000 ~ b}$ & $0.99990_{-0.00872}^{+0.00000 ~ b}$ & $0.99990_{-0.00838}^{+0.00000 ~ b}$ & $0.99990_{-0.00545}^{+0.00000 ~ b ~}$ & $0.99950_{-0.00348}^{+0.00013 \mathrm{~b}}$ \\
\hline 2 & KERRBB2 & $\dot{M}$ & $0.119 \pm 0.013$ & $0.121 \pm 0.013$ & $0.116 \pm 0.012$ & $0.108 \pm 0.007$ & $0.113 \pm 0.005$ \\
\hline 3 & Const & $\cdots$ & $0.7819 \pm 0.0074$ & $0.6257 \pm 0.0075$ & $0.7534 \pm 0.0073$ & $0.7566 \pm 0.0055$ & $0.7518 \pm 0.0065$ \\
\hline 5 & SIMPLR & $\Gamma$ & $2.4438 \pm 0.0094$ & $2.4906 \pm 0.0098$ & $2.5753 \pm 0.0094$ & $2.4662 \pm 0.0081$ & $2.5751 \pm 0.0081$ \\
\hline 6 & SIMPLR & $f_{\mathrm{s}}$ & $0.1347 \pm 0.0027$ & $0.1783 \pm 0.0034$ & $0.1924 \pm 0.0033$ & $0.1022 \pm 0.0015$ & $0.1195 \pm 0.0016$ \\
\hline 7 & KERRDISK & $E_{\mathrm{L}}$ & $6.571 \pm 0.036$ & $6.482 \pm 0.059$ & $6.446 \pm 0.048$ & $6.560 \pm 0.032$ & $6.466 \pm 0.036$ \\
\hline 10 & KERRDISK & EW & 0.283 & 0.238 & 0.211 & 0.292 & 0.228 \\
\hline 11 & IREFLECT $^{\mathrm{c}}$ & {$[\mathrm{Fe}]$} & $5.4269 \pm 0.4637$ & $3.9534 \pm 0.2995$ & $4.3540 \pm 0.3139$ & $4.7329 \pm 0.3721$ & $3.7402 \pm 0.2688$ \\
\hline 12 & IREFLECT & $\xi$ & $140.0 \pm 13.2$ & $94.3 \pm 11.6$ & $87.9 \pm 8.7$ & $166.0 \pm 13.2$ & $121.6 \pm 8.7$ \\
\hline 13 & & $\chi_{v}^{2}$ & $0.95(595 / 628)$ & $1.02(587 / 573)$ & $0.97(605 / 625)$ & $1.20(890 / 745)$ & $1.12(1119 / 998)$ \\
\hline 14 & & $f$ & 1.60 & 1.62 & 1.62 & 1.61 & 1.61 \\
\hline 15 & & $L / L_{\text {Edd }}$ & 0.022 & 0.022 & 0.022 & 0.020 & 0.019 \\
\hline
\end{tabular}

Notes.

${ }^{a}$ For the model components given, the parameters from top to bottom are: (1) spin parameter; (2) mass accretion rate in units of $10^{18} \mathrm{~g} \mathrm{~s}^{-1}$; (3) detector normalization constant relative to RXTE PCU2; (4) hydrogen column density in units of $10^{22} \mathrm{~cm}^{-2}$; (5) photon power-law index $\Gamma$; (6) scattering fraction $f_{\mathrm{s}}$; (7) central line energy in keV; (8) emissivity index $q$; (9) line flux in units of photons $\mathrm{cm}^{-2} \mathrm{~s}^{-1}$; (10) equivalent width of line in keV; (11) iron abundance relative to solar; (12) ionization parameter $\xi$; (13) Reduced chi-square, total chi-square, and degrees of freedom, respectively; (14) spectral hardening factor $f$; and (15) Eddington-scaled disk luminosity, where $L_{\mathrm{Edd}} \approx 1.9 \times 10^{39} \mathrm{ergs}^{-1}$ for Cygnus X-1. The confidence levels on the uncertainties quoted here and throughout the paper, unless indicated otherwise, are $1 \sigma$.

b Although the physical limit on the spin parameter for disk accretion is $a_{*} \approx 0.998$ (Thorne 1974), the formal maximum value for the KERRBB2 model is 0.9999 . The errors quoted here were computed using the command error in XSPEC and are the uncertainties due to counting statistics only.

${ }^{c}$ The scaling factor $s$ in the model IREFLECT was set to unity for all fits (see text).

${ }^{\mathrm{d}}$ Final adopted values for the spin parameter and their uncertainties. The $1 \sigma$ uncertainties are estimated based on the $3 \sigma$ lower limits on $a_{*}$ shown in Figure 5 . These results fold in the uncertainties in $D, M, i$, and the absolute flux calibration via our Monte Carlo analysis (see Section 6).

Table 3

Fit Results for Observations 2-4: Spectra S6-S10

\begin{tabular}{|c|c|c|c|c|c|c|c|}
\hline Number & Model & Parameter & S6 & S7 & S8 & S9 & S10 \\
\hline 1 & KERRBB2 & $a_{*}$ & $0.99990_{-0.00922}^{+0.00000}$ & $0.97177_{-0.00450}^{+0.00938}$ & $0.99990_{-0.00520}^{+0.00000}$ & $0.99988_{-0.00546}^{+0.00001}$ & $\overline{0.99990_{-0.00842}^{+0.00000}}$ \\
\hline 2 & KERRBB2 & $\dot{M}$ & $0.115 \pm 0.013$ & $0.194 \pm 0.008$ & $0.113 \pm 0.007$ & $0.128 \pm 0.008$ & $0.108 \pm 0.011$ \\
\hline 3 & Const & $\ldots$ & $0.8989 \pm 0.0379$ & $0.7259 \pm 0.0797$ & $1.2432 \pm 0.0116$ & $1.3873 \pm 0.0085$ & $1.8046 \pm 0.0191$ \\
\hline 4 & TBABS & $N_{\mathrm{H}}$ & $0.7148 \pm 0.0103$ & $0.7241 \pm 0.0182$ & $0.7875 \pm 0.0062$ & $0.7527 \pm 0.0054$ & $0.7911 \pm 0.0098$ \\
\hline 5 & SIMPLR & $\Gamma$ & $2.6976 \pm 0.0062$ & $2.7430 \pm 0.0079$ & $2.6248 \pm 0.0088$ & $2.6649 \pm 0.0071$ & $3.0264 \pm 0.0162$ \\
\hline 6 & SIMPLR & $f_{\mathrm{s}}$ & $0.2359 \pm 0.0041$ & $0.2942 \pm 0.0058$ & $0.2927 \pm 0.0038$ & $0.4800 \pm 0.0111$ & $0.3118 \pm 0.0077$ \\
\hline 7 & KERRDISK & $E_{\mathrm{L}}$ & $6.514 \pm 0.026$ & $6.531 \pm 0.036$ & $6.545 \pm 0.072$ & $6.516 \pm 0.046$ & $6.539 \pm 0.049$ \\
\hline 8 & KERRDISK & $q$ & $2.293 \pm 0.049$ & $2.152 \pm 0.081$ & $2.923 \pm 0.061$ & $2.467 \pm 0.058$ & $2.233 \pm 0.107$ \\
\hline 9 & KERRDISK & $N_{\mathrm{L}}$ & $0.017 \pm 0.001$ & $0.017 \pm 0.001$ & $0.016 \pm 0.002$ & $0.023 \pm 0.001$ & $0.011 \pm 0.001$ \\
\hline 10 & KERRDISK & EW & 0.190 & 0.141 & 0.176 & 0.146 & 0.187 \\
\hline 11 & IREFLECT & {$[\mathrm{Fe}]$} & $4.0832 \pm 0.1660$ & $3.4452 \pm 0.1602$ & $4.2666 \pm 0.4452$ & $3.2580 \pm 0.1721$ & $1.3208 \pm 0.1606$ \\
\hline 12 & IREFLECT & $\xi$ & $74.3 \pm 5.2$ & $42.8 \pm 5.0$ & $220.4 \pm 24.9$ & $66.5 \pm 6.2$ & $82.3 \pm 14.6$ \\
\hline 13 & & $\chi_{v}^{2}$ & $1.40(491 / 352)$ & $1.61(323 / 201)$ & $1.37(484 / 353)$ & $1.54(612 / 399)$ & $1.24(416 / 337)$ \\
\hline 14 & & $f$ & 1.60 & 1.59 & 1.59 & 1.60 & 1.59 \\
\hline 15 & & $L / L_{\mathrm{Edd}}$ & 0.021 & 0.021 & 0.021 & 0.024 & 0.020 \\
\hline 16 & Adopted & $a_{*}$ & $0.99990_{-0.00597}^{+0.00000}$ & $\cdots$ & $\cdots$ & $\cdots$ & $\cdots$ \\
\hline
\end{tabular}

Note. ${ }^{\text {a }}$ Layout and parameter definitions are exactly the same as for Table 2.

\section{RESULTS}

In this section, we present results with the key input parameters fixed at their fiducial values: $D=1.86 \mathrm{kpc}, M=14.8 M_{\odot}$, and $i=27.1 \mathrm{deg}$ (Reid et al. 2011; Orosz et al. 2011). The fit results for all ten spectra, S1-S10, are summarized in Tables 2 and 3 .

Before broadly discussing the results, we focus on the value of the scattering fraction, $f_{\mathrm{s}}$ (Tables 2 and 3 ), and we strictly follow the data selection criterion $f_{\mathrm{s}} \lesssim 25 \%$ (Steiner et al. 2009a). Therefore, we henceforth consider only the six spectra S1-S6 for which $f_{\mathrm{s}} \leqslant 24 \%$, and we disregard the remaining spectra (S7-S10).

Before focusing solely on spectra S1-S6, however, we note that the results for the rejected spectra are, in detail, consistent with those of the selected spectra. The most notable difference is the depressed value of $a_{*}$ for S7 (0.972 versus 0.999 for the other nine spectra); however, note the poor fit achieved for S7 $\left(\chi_{v}^{2} /\right.$ dof $=1.61 / 201$ versus a mean value of 1.11 for S1-S6). Meanwhile, a comparison of the mean values of the parameters 
for spectra S1-S6 with their corresponding mean values for spectra S7-S10 shows that in most cases the mean values differ by $\lesssim 2 \%$ (Tables 2 and 3). The two notable exceptions (apart from of course the scattering fraction) are the steeper powerlaw slope $(\Delta \Gamma=0.084)$ and significantly weaker Fe line for the four rejected spectra. Finally, we note that the values of $f_{\mathrm{s}}$ for three of the rejected spectra (S7, S8, and S10) are very nearly the same as for the two inferior spectra used in GOU11 (SP2 and SP3), namely, $f_{\mathrm{s}} \approx 30 \%$.

We now direct our attention hereafter solely to spectra S1-S6 with values of $f_{\mathrm{s}}=10 \%-24 \%$. The fits are all good, with $\chi_{v}^{2} /$ dof ranging for $\mathrm{S} 1$ from $0.95 / 628$ to $1.40 / 491$ for S6. The spin parameter is very high and is pegged at the $a_{*}=0.9999$ limit of the KERRBB2 model (McClintock et al. 2013), which is the principal result of this section.

The luminosity of the disk component is low and uniform, $L / L_{\text {Edd }}=1.9 \%-2.2 \%$, and it easily meets a key data selection criterion for successful application of the continuum-fitting method, namely, $L / L_{\text {Edd }}<30 \%$ (McClintock et al. 2006, 2013). Correspondingly, the disk is expected to be geometrically thin at all radii $(h / r<0.05$; see Penna et al. 2010; Kulkarni et al. 2011; Zhu et al. 2012). Meanwhile, the spectral hardening factor $f$ is well determined $(f \approx 1.6)$ because the disk luminosity is sufficiently high.

The column density is statistically well determined with uncertainties of only $1 \%-2 \%,{ }^{14}$ while it varies by $3.3 \%$ (std. deviation; $N=6$ ). The variability is unsurprising since $N_{\mathrm{H}}$ varies by several percent for all three well-studied supergiant black-hole binaries: M33 X-7 (Liu et al. 2008), Cygnus X-1 (Hanke et al. 2009), and LMC X-1 (Hanke et al. 2010). The power-law slope is well determined and quite stable, $\Gamma=2.52 \pm 0.12$ (std. deviation; $N=6$ ), and its value is the expected one for the steep power-law state $(\Gamma>2.4$; Remillard $\&$ McClintock 2006). The ionization parameter is modest and in the range $\xi \approx 70-170$.

\section{ROBUSTNESS OF SPIN ESTIMATES}

In GOU11, we discuss many factors that might affect our principal result, namely, the extreme spin of Cygnus $\mathrm{X}-1$; we find that none of them are significant. Here, we review these matters briefly. For further details, see Sections 5 and 7 in GOU11, and see also Section 5 in McClintock et al. (2013). Sections 5.5 and 5.6 below are wholly new and discuss our adopted reflection model in relation to the recently released reflection model XILLVER (García et al. 2013). Section 5.2 on pileup and Section 5.8 on the effect of dust scattering are likewise new.

\subsection{Errors from the Novikov-Thorne Model}

The accuracy of continuum-fitting results ultimately depends on the reliability of the Novikov-Thorne model. The key assumption of this model is that the torque, and hence the flux, vanishes at the ISCO (Shafee et al. 2008; Penna et al. 2010). The effects of this approximation on spin measurements have been quantitatively investigated via general relativistic magnetohydrodynamic (GRMHD) simulations of thin disks by several authors (Noble et al. 2011; Kulkarni et al. 2011; Zhu et al. 2012). The general consensus is that the zero-torque

\footnotetext{
14 The average value of $N_{\mathrm{H}},(0.754 \pm 0.016) \times 10^{22} \mathrm{~cm}^{-2}$, agrees very well with the values derived from the $21 \mathrm{~cm}$ line in the direction of Cygnus X-1, which is $N_{\mathrm{H}}=0.721 \times 10^{22} \mathrm{~cm}^{-2}$, a weighted average from both LAB and DL maps (Kalberla et al. 2005; Dickey \& Lockman 1990).
}

approximation introduces uncertainties in spin estimates of around $\Delta a_{*} \sim 0.1$ for low spin values $\left(a_{*}<0.5\right)$ and much smaller errors as $a_{*} \rightarrow 1$. These error estimates, which are for geometrically thin disks $\left(H / R \approx 0.05\right.$, or $\left.L / L_{\text {Edd }} \sim 0.35\right)$ are in practice less than the observational errors in the parameters $D, M$, and $i$. For more details concerning the Novikov-Thorne model and a discussion of other sources of model errors, see Section 5 in McClintock et al. (2013).

\subsection{Effects of Pileup}

We estimated the pileup fraction for each spectrum from Equation (2) in the Chandra ABC Guide to Pileup ${ }^{15}$ using the observed photon flux as the input. There can be no clear-cut prescription for what level of pileup is acceptable because its effects depend in complex ways on detector performance and science goals. The guideline on pileup for Chandra data stated in the Chandra Proposer's Observatory Guide (Section 6.15.12) ${ }^{16}$ is: "If one's scientific objective demands precise flux calibration, then the pileup fraction should probably be kept well below $10 \%$." A specific concern for this paper is that pileup effects, which tend to harden a continuum spectrum, might significantly boost the value of the spin parameter. We find this not to be the case, as we now discuss.

\subsubsection{Chandra CC Mode}

For the five CC-mode spectra (S1-S5), the effects of pileup are small, $<1.5 \%$ over the full fitting range of $0.8-8.0 \mathrm{keV}$ (Figure 4), because of the gratings and the nominal $2.85 \mathrm{~ms}$ frame time. ${ }^{17}$ We nevertheless made two tests to assess the effects of pileup, both of which show that they are negligible. First, we refitted the five spectra ignoring the Chandra data above $2.0 \mathrm{keV}$ (i.e., the data that determine $\Gamma$ ), and we found that the values of the key parameter, $a_{*}$, remained unchanged and pegged at the physical limit, while $\Gamma$ and the scattering fraction in all cases changed by less than $1.1 \%$ and $4.0 \%$, respectively. The small change in $\Gamma$ is as expected, since the number of Chandra counts in the spectrum above $2 \mathrm{keV}$ is only $\approx 1 \%$ of the $R X T E$ counts.

Second, we performed a MARX simulation ${ }^{18}$ to quantitatively estimate how the pileup fraction affects the power-law component for a single Chandra spectrum (i.e., excluding its companion RXTE spectrum). Because MARX does not support the $\mathrm{CC}$ mode we relied on simulations of TE-mode data. We simulated a parent TE-mode spectrum using parameters that describe a typical Cygnus X-1 spectrum. We then used the simulated spectrum to generate four fake spectra with pileup fractions (at peak flux) of 1.5\%, 3\%, 5\%, and 10\%. We fitted these spectra using our nonrelativistic model NR3 (GOU11; the power-law component is poorly constrained for the relativistic model), excluding in this case the reflection component PEXRIV, and we compared the results to the results obtained by fitting the parent spectrum $\left(\Gamma=2.963, f_{\mathrm{s}}=0.187\right.$, $\left.T_{\text {in }}=0.423, N_{T}=91.28\right)$. The photon index $\Gamma$ increased, respectively, by $0.8 \%, 2.2 \%, 2.1 \%$, and $6.4 \%$, and the fractional change in the scattering fraction was $6.7 \%, 15 \%, 22 \%$, and $59 \%$. Meanwhile, concerning the thermal component, the disk temperature decreased, respectively, by $0.1 \%, 0.5 \%, 0.3 \%$,

\footnotetext{
15 http://cxc.harvard.edu/ciao/download/doc/pileup_abc.pdf

16 http://cxc.harvard.edu/proposer/POG/

17 A frame time of $9 \mathrm{~ms}$ was conservatively assumed in making the pileup estimate.

18 http://space.mit.edu/CXC/MARX/
} 


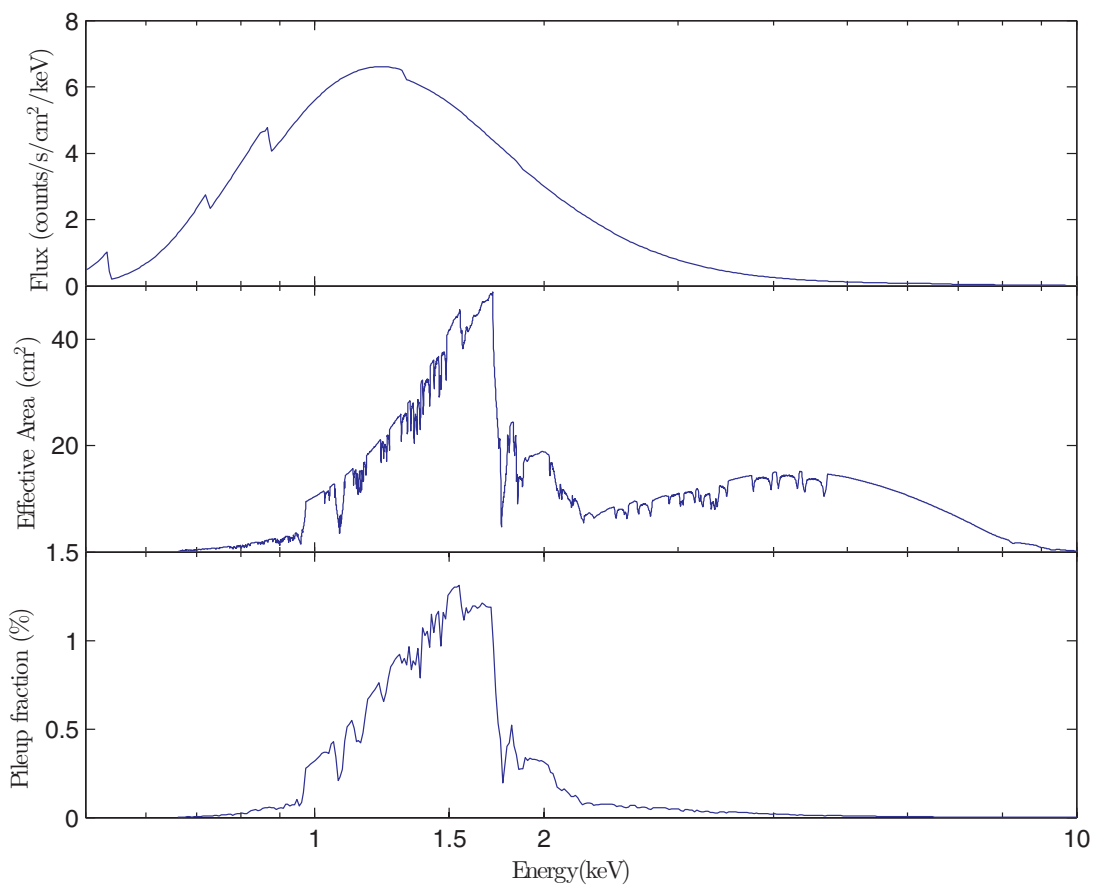

Figure 4. Estimate of the pileup fraction (bottom) for a nominal spectrum of Cygnus X-1 (top) and the HEG(-1) effective area in CC mode (middle). The estimate was computed using Equation (2) in The Chandra ABC Guide to Pileup assuming conservatively (1) that the frame integration time is three times the 2.85 ms default frame time and (2) that the grade migration parameter $\alpha=1.0$ (i.e., the probability that a piled event is retained is unity).

(A color version of this figure is available in the online journal.)

and $2.3 \%$, while the corresponding normalization constant decreased by $2.2 \%, 3.6 \%, 8.1 \%$, and $9.9 \%$. Because $R_{\text {in }}$ is proportional to the square root of the disk normalization, the fractional change in $R_{\text {in }}$ is even smaller, decreasing, respectively, by $1.1 \%, 1.8 \%, 4 \%$, and $5 \%$. We conclude therefore that the spin is likely to be only very moderately overestimated. These results give reasonable assurance that our fit results (Tables 2 and 3 ) are negligibly affected by pileup, given that the peak pileup fraction for the five CC-mode spectra is $<1.5 \%$ and for the TE-mode streak spectrum is $<3 \%$ (see below).

\subsubsection{Chandra TE Mode}

Our two TE-mode spectra (S6 and S7) suffer more than the CC-mode spectra from the effects of pileup, especially the HEG and MEG components of the spectrum. We estimated the pileup fraction using the kernel PILEUP in ISIS, whose mathematical formulation is also Equation (2) in the Chandra $\mathrm{ABC}$ Guide to Pileup. The pileup fraction for the streak spectrum is $<3 \%$ over our entire fitting range of $0.5-10 \mathrm{keV}$. In order to ensure a pileup fraction of $<5 \%$ for the MEG and HEG spectra, we only used data in two restricted energy ranges: $0.7-0.9 \mathrm{keV}$ and 7.0-10.0 keV. As a test for the effects of pileup, we refitted spectrum S6 excluding the MEG and HEG data. The largest effect on the fit parameters was a $0.7 \sigma$ change in the column density, which decreased from $(0.714 \pm 0.010) \times 10^{22} \mathrm{~cm}^{-2}$ to $(0.698 \pm 0.022) \times 10^{22} \mathrm{~cm}^{-2}$. The changes in the best-fit values of the other parameters are much less than $1 \%$.

\subsubsection{Swift}

The three X-Ray Telescope (XRT) WT-mode spectra (S8-S10) have the same format as the Chandra CC-mode spectra; i.e., they are collapsed one-dimensional strips rather than images. We reduced the effects of pileup by excluding the central $15 \times 20$ pixel region (i.e., a 20 pixel wide swath extending 15 pixels along the image strip), a choice validated by Romano et al. (2006). These authors performed pileup tests with the excluded region ranging from 0 pixels to 15 pixels (i.e., from $0 \times 20$ pixels to $15 \times 20$ pixels) and for five levels of source intensity. They found no pileup effects (i.e., spectral distortion) for count rates in the range $0-100$ counts $\mathrm{s}^{-1}$, and only moderate effects in the range 100-300 counts s ${ }^{-1}$. In our case, we therefore expect minimal pileup effects because our count rate (with the central region excluded) is only $\approx 120$ counts s $^{-1}$ after the exclusion. Nevertheless, we performed one additional test: we refitted spectrum S8 ignoring the XRT data above $3 \mathrm{keV}$ (while fitting jointly with the RXTE data, as before) and found our fit results to be the same as those reported in Table 3 .

\subsection{Effect of Iron Line and Edges}

In GOU11, we showed that the Fe line and other reflection features in soft-state spectra of Cygnus X-1 are cosmetic and have a negligible effect on the continuum-fitting measurement of spin. Specifically, we refitted the three spectra considered in GOU11 excluding the 5.0-10.0 keV band and the Fe-line component KERRDISK. This removed the energy range covering the $\mathrm{Fe} \mathrm{K} \alpha$ line and edge as well as a feature in the residuals near $9 \mathrm{keV} .{ }^{19}$ We found that our spin results were essentially unchanged, as expected given the modest equivalent widths of these features and the relative faintness of the reflected component (see Section 5.6 and Figure 3).

\subsection{Effect of Extending the Bandwidth from $45 \mathrm{keV}$ to $150 \mathrm{keV}$}

In Section 5.2 of GOU11, we showed that the energy coverage of the PCA, which extends to $45 \mathrm{keV}$, is sufficient to adequately

\footnotetext{
19 This feature results from the imperfect performance of IREFLECT/PEXRIV (Section 3), the reflection model we employ. The limitations of this model, which are well known (Ross et al. 1999; García et al. 2013), are discussed in Section 5.6, while the model's marginal performance near the Fe edge is illustrated in Figure 5.
} 
constrain both the power-law and reflection components. We did this by refitting one spectrum including RXTE HEXTE data, which cover the range $20 \mathrm{keV}$ to $150 \mathrm{keV}$. This result is not surprising since coverage to $45 \mathrm{keV}$ is more than adequate to determine the power-law component and the reflection component is decreasing rapidly at $45 \mathrm{keV}$ (Figure 3).

\subsection{Effect of using a Different Reflection Model}

As in Section 5.3 in GOU11, we replaced our reflection component KERRCONV $\otimes$ IREFLECT $\otimes$ SIMPLC+KERRDISK with KERRCONV $\otimes$ REFLIONX (Ross \& Fabian 2005), which is widely used in measuring spin via the $\mathrm{Fe} \mathrm{K} \alpha$ line. As in GOU11, we again find that the effects on the spin parameter are essentially nil. More recently, a new and improved reflection model XILLVER has become available (García et al. 2013). This version of XILLVER (like REFLIONX) is intended for use when the thermal disk flux is faint compared to the incident power-law flux, and it is therefore not well-suited to our case. Nevertheless, as with REFLIONX, we performed a test by replacing our reflection component with KERRCONV $\otimes$ XILLVER. The fits are poorer with reduced chi-square ranging from 1.9 to 2.2 for $\mathrm{S} 1-\mathrm{S} 5$, but the effects on the spin parameter were again found to be negligible (less than $0.2 \%$ ).

\subsection{On the Accuracy of our Adopted Reflection Model}

In computing the reflected component, we rely on IREFLECT, which generates a spectrum containing sharp absorption features and no emission lines. Figure 20 in García et al. (2013) shows that (ignoring line emission) IREFLECT/PEXRIV is a good approximation to the sophisticated model XILLVER at low ionization, $\xi=1$ (left panel), while it is a very poor approximation at high ionization, $\xi=10^{3}$ (right panel). In Figure 5, we show that for an intermediate case, $\xi \sim 10^{2}$, which corresponds to the moderately ionized disk of Cygnus X-1 (see Tables 2 and 3), IREFLECT/PEXRIV is in reasonable agreement with XILLVER. Considering further that the peak reflected flux is $\approx 25$ times fainter than the peak thermal flux (Figure 3), it is not surprising that our estimate of spin is insensitive to the choice of reflection model.

In all the fits, we have fixed the disk temperature in the reflection model at $6.0 \times 10^{6} \mathrm{~K}$, which corresponds to $0.52 \mathrm{keV}$. The disk temperature is quite constant at this value for spectra S1-S6 and the three spectra in GOU11 (see Section 3). Meanwhile, increasing the disk temperature by $50 \%$ to $9.0 \times 10^{6} \mathrm{~K}$ or halving it has a negligible effect on the spin and other key parameters (apart from the ionization parameter).

\subsection{Effect of Varying the Viscosity Parameter and Metallicity}

Reanalyzing the data using $\alpha=0.01$ instead of our adopted value of $\alpha=0.1$ has a very slight effect on our results, and doing so only increases the already extreme value of spin. The effects of varying metallicity are likewise very small, whether one grossly decreases its value to a tenth solar or considers the suprasolar values implied by the IREFLECT fits (Tables 1 and 2). In the former/latter case, the spin is depressed/increased, but only very slightly (see Section 5.4 in GOU11). An analysis of high resolution optical spectra of the donor star indicates that Fe is somewhat overabundant relative to solar (Karitskaya et al. 2007).

\subsection{Effect of a Warm Absorber}

In determining the spins of supermassive black holes via the $\mathrm{Fe} \mathrm{K} \alpha$ method, careful modeling of absorption by intervening

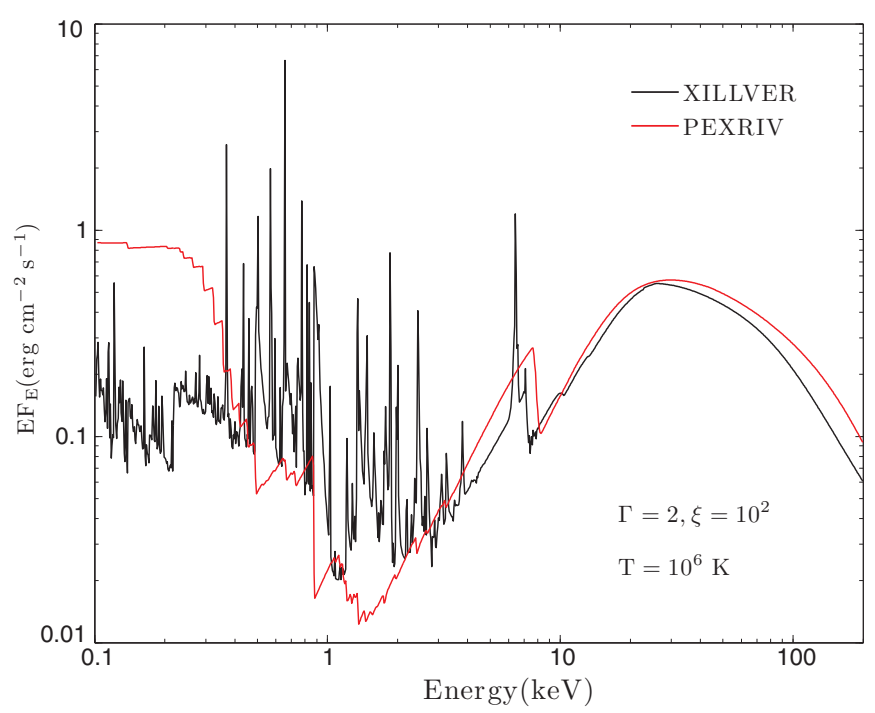

Figure 5. Comparison of reflected spectra computed using the advanced model XILLVER (black curve with emission lines) and using PEXRIV (red curve) for a power-law spectrum with photon index $\Gamma=2$ incident on an optically thick slab of gas; the ionization parameter in this example, $\xi=100$, is a good match to the values observed for Cygnus X-1. This figure was computed by J. Garcia in precisely the same way as the pair of figures shown in Figure 20 in García et al. (2013). The disk temperature in the PEXRIV model is set to its maximum possible value, $T=10^{6} \mathrm{~K}$; the high-energy cutoff is $300 \mathrm{keV}$; and the abundances are assumed to be solar. The obvious discrepancy between the models in the vicinity of the Fe $\mathrm{K}$ complex is the origin of the residual feature near $9 \mathrm{keV}$ that is apparent in the lower panel of Figure 3. For reasons discussed in Section 5.3, this feature does not affect our estimate of spin. The large discrepancies between the two models at $E<0.4 \mathrm{keV}$ have no bearing on our results because the lower bound of our fitting range is $0.5 \mathrm{keV}$ (Table 1). (A color version of this figure is available in the online journal.)

warm gas is usually crucial (e.g., Brenneman \& Reynolds 2006). However, we have shown, via a continuum-fitting analysis of Chandra HETG spectra, that the effect of warm absorbers is unimportant in estimating the spin of Cygnus X-1 (see Section 7.6 in GOU11).

\subsection{Effect of Dust Scattering}

The dust scattering halo of Cygnus X-1 (e.g., Xiang et al. 2011) has an effect on the source spectrum that is equivalent to direct absorption. In order to assess the effects of dust scattering on our results, we used the only relevant model that is presently available in XSPEC, namely, DUST. The model assumes that the source flux is scattered into a uniform disk whose size and total flux vary, respectively, as $1 / E$ and $1 / E^{2}$. The simple model DUST is a good approximation to more accurate models (e.g., Weingartner \& Draine 2001) at energies in the bandpass of interest, namely, $E>0.8 \mathrm{keV}$ (Table 1).

We reanalyzed spectra $\mathrm{S} 1-\mathrm{S} 5$ as before, but this time we included the multiplicative model component DUST. The model has two parameters that specify at $1 \mathrm{keV}$ (1) the fraction of photons scattered by dust grains, and (2) the size of the halo in units of the detector beam size. If both parameters are allowed to vary, neither can be constrained. We therefore initially fixed the scattering parameter to 0.17 , which was obtained by extrapolating the value 0.12 at $1.2 \mathrm{keV}$ given by Predehl \& Schmitt (1995, see their Figure 10). The results obtained for the key parameters $a_{*}$ and $f_{\mathrm{s}}$ for each of the five spectra are essentially identical to those that appear in Table 2, although the column density $N_{\mathrm{H}}$ is reduced by $\approx 13 \%$. Even if one increases the dust scattering parameter from 0.17 to 0.3 , the values of $a_{*}$ 


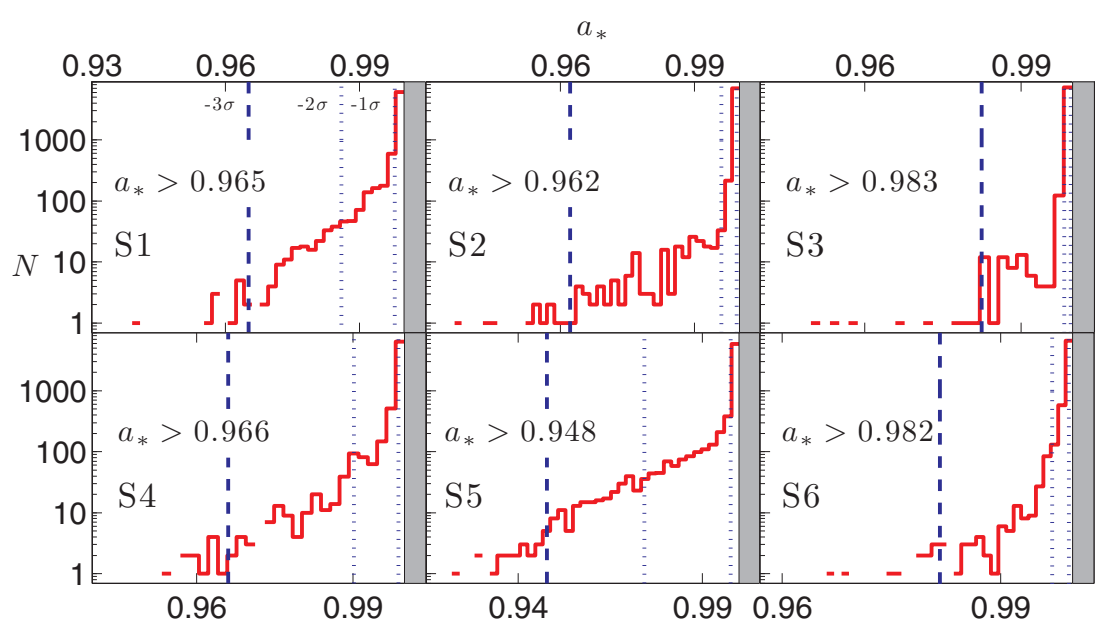

Figure 6. Histograms resulting from the Monte Carlo analysis for 9000 parameter sets (per spectrum) for all selected spectra with $f_{\mathrm{s}}<25 \%$. The level of confidence on the lower limits on $a_{*}$ are $99.7 \%(3 \sigma)$.

(A color version of this figure is available in the online journal.)

and $f_{\mathrm{s}}$ are essentially unchanged, while in this case $N_{\mathrm{H}}$ is reduced by $\approx 25 \%$. We conclude that the effects of dust scattering are unimportant.

\subsection{Effect of a Possible Spin-Orbit Misalignment}

In Section 7.4 in GOU11, we considered a principal source of uncertainty in the continuum-fitting method, namely, whether the black hole's spin axis and the inner disk will align with the orbital plane. If, as some evidence suggests, the persistent supergiant systems are formed by direct, kickless collapse (Mirabel \& Rodrigues 2003; Reid et al. 2011), then spin-orbit alignment would be expected for these systems. (For full discussions on the topic of spin-orbit alignment, see Section 1 in Steiner et al. 2012, and Section 5.4 in McClintock et al. 2013). In any case, as we demonstrate for Cygnus X-1 in Figure 5 in GOU11, even for a misalignment angle as large as, e.g., $16 \mathrm{deg}$ the spin parameter is still $>0.95$ (ignoring the uncertainties in $D, M$, and $i$ ).

\section{COMPREHENSIVE ERROR ANALYSIS}

The dominant error in all continuum-fitting measurements of spin is attributable to the observational uncertainties in the source distance, black hole mass, and disk inclination. For Cygnus X-1, we have determined accurate values for these quantities: $D=1.86_{-0.11}^{+0.12} \mathrm{kpc}$ (Reid et al. 2011), $M=$ $14.8 \pm 1.0 M_{\odot}$, and $i=27.1 \pm 0.8 \mathrm{deg}$ (Orosz et al. 2011).

Quite generally, even the uncertainties in the analytic Novikov-Thorne model are significantly less important than the uncertainties in $D, M$, and $i$, as has been shown via GRMHD simulations of thin accretion disks (Section 5.1). The model errors in the case of Cygnus X-1 are very small because the black hole's spin is extreme and the disk's luminosity is low, only $\approx 2 \%$ of the Eddington limit. Kulkarni et al. (2011) have shown via a detailed analysis that for an inclination of $30 \mathrm{deg}$ (closely approximating Cygnus X-1's $27 \mathrm{deg}$ inclination) the Novikov-Thorne model overestimates the spin parameter by only $\Delta a_{*} \approx 0.006$ for spin parameters in the range $0.90-0.98$.

The contribution to the uncertainty in the spin of Cygnus X-1 due to the uncertainty in the absolute calibration of the flux is about the same as that due to the $6 \%$ uncertainty in the distance.
We therefore include in our error budget a $10 \%$ uncertainty in flux (Toor $\&$ Seward 1974) by inflating the uncertainty in $D$ by the method described in GOU11. The final error we report for $a_{*}$ therefore includes the uncertainty in the absolute flux calibration as well as the uncertainties in $D, M$, and $i$. Collectively, the uncertainties in these four quantities completely dominate the error budget. (Other, smaller sources of error are discussed in detail in Appendix A and Section 5 in Steiner et al. 2011, and Section 5 in McClintock et al. 2013.)

Following precisely the same procedures described in Section 6 of GOU11, we determined the error in $a_{*}$ due to the combined uncertainties in $D, M$, and $i$ via Monte Carlo simulations. Figure 6 shows the resultant spin histograms for our six spectra and displays for each spectrum the corresponding lower limits on $a_{*}$ at a $3 \sigma$ level of confidence.

Were we to use these six limits to derive a joint constraint on spin, it would be more stringent than any one of the individual limits. We choose instead the conservative approach of adopting the most constraining single limit for our final result, namely, the limit for spectrum S3. We therefore conclude that $a_{*}>0.983$ at the $3 \sigma$ level of confidence. ${ }^{20}$

We note the following two caveats. First, we assume that the spin of the black hole is approximately aligned with the angular momentum vector of the binary (Section 5.10). Second, we assume that the asynchronous dynamical model is correct (see Section 7.3 in GOU11).

\section{DISCUSSION}

We first discuss three spin estimates for Cygnus X-1 made using the Fe-line method, which provide support for an extreme value of spin. We then relate Cygnus X-1 to the other members of its distinctive class of black-hole X-ray sources that are persistently bright.

\subsection{Measurement of Spin via the Fe-K/Reflection Method}

Three recent measurements of the spin of Cygnus X-1 obtained using X-ray reflection spectroscopy, aka the Fe line

\footnotetext{
20 In GOU11, we conservatively adopted the limit $a_{*}>0.95$ obtained for SP1 as our final result because it was the only one of the three spectra whose scattering fraction was $<25 \%$.
} 
Table 4

Data for Three Persistent Black Hole Binaries

\begin{tabular}{lcccccc}
\hline \hline Source $^{\mathrm{a}}$ & $a_{*}$ & $M\left(M_{\odot}\right)$ & $M_{2}\left(M_{\odot}\right)$ & $P($ days $)$ & $L / L_{\text {Edd }}$ & References \\
\hline Cygnus X-1 & $>0.983$ & $14.8 \pm 1.0$ & $19.2 \pm 1.9$ & 5.60 & 0.02 & This work; Orosz et al. 2011 \\
LMC X-1 & $0.92_{-0.07}^{+0.05}$ & $10.9 \pm 1.4$ & $31.8 \pm 3.5$ & 3.91 & 0.16 & Gou et al. 2009; Orosz et al. 2009 \\
M33 X-7 & $0.84 \pm 0.05$ & $15.7 \pm 1.5$ & $70.0 \pm 6.0$ & 3.45 & 0.09 & Liu et al. 2008; Orosz et al. 2007 \\
\hline
\end{tabular}

Note. ${ }^{a}$ From left to right, the parameters are, respectively, spin parameter, black hole mass, mass of the secondary, orbital period, and the Eddington-scaled disk luminosity.

method (Reynolds 2013), support a high or extreme value of spin.

Duro et al. (2011) report $a_{*}=0.88_{-0.11}^{+0.07}$. Their provisional result is based on an analysis of a single simultaneous observation made using XMM-Newton and RXTE. A limitation of their result is that it depends on assuming a single, fixed value of 3 for the emissivity index $q$, which is a canonical value. That is, they assume that the intensity of the flux irradiating the disk varies with radius as $r^{-3}$. When they allow $q$ to vary freely, both the spin parameter and emissivity index are poorly constrained (see their Table 1). In short, their data are unable to determine both the profile of the illuminating radiation and the spin.

The result of Duro et al. (2011) is superseded by that of Fabian et al. (2012) who report $a_{*}=0.97_{-0.02}^{+0.014}$. This result is based on an analysis of a single hard-state $S u z a k u$ spectrum. Fabian et al. describe this spectrum as "an average data set" (from a collection of 20 similar spectra) and report that consistent results were obtained for other data sets. The fit over a $1-500 \mathrm{keV}$ band gives precise results for a three-parameter, broken power-law model of the radial profile of the irradiating flux: Inside the break radius $\left(R_{\text {break }}=4.0 \pm 1.1 G M / c^{2}\right), q>6.8$, and outside $q=2.75 \pm 0.15$.

Most recently, Tomsick et al. (2014) fitted the Fe-K $\alpha$ line using Suzaku and NuSTAR data. Cygnus X-1 was in the soft state. Their best-fitting model gives $a_{*}=0.9882 \pm 0.0009$ ( $90 \%$ confidence level) and all the models that provided a good fit to the spectrum indicate a rapidly rotating black hole with $a_{*}>0.83$.

A strength of this work relative to prior studies of Cyg X-1's spin (including our first paper, GOU11) is the considerable attention we give here to assessing the effects of a wide range of systematic errors. In doing so, and from a wider breadth of data, our work supplies the strongest evidence for Cyg X-1's extreme spin, confirming the prior leading measurements by GOU11 and Fabian et al. (2012).

Earlier, Miller et al. (2009) reported a near-zero spin for Cygnus X-1, $a_{*}=0.05 \pm 0.01$, based on an analysis of two XMM-Newton spectra. Neither Fabian et al. (2012) nor Duro et al. (2011) offer an explanation for this glaring discrepancy. However, recently an explanation was suggested for the nearzero spin reported by Miller et al. in terms of pileup effects (see Section 4.3 in Reynolds 2013). This example shows that measurements of spin in the literature can be grossly affected by systematic effects, which should be carefully considered in assessing the reliability of spin results.

\subsection{Cygnus X-1 and the Other Persistent Black Hole Systems}

There are five dynamically established black-hole binaries containing wind-fed black holes and O-supergiant or Wolf-Rayet companions (Özel et al. 2010; McClintock et al. 2013); these systems are persistently X-ray bright. In the following, we do not consider the two systems with Wolf-Rayet companions, IC $10 \mathrm{X}-1$ and NGC $300 \mathrm{X}-1$, because the masses of their black holes are very uncertain and their spins have not been estimated. By contrast, the three remaining systemsCygnus X-1, LMC X-1, and M33 X-7-have well-determined values of both mass and spin. These fundamental data, which provide a complete description of these three black holes, appear in the two leftmost columns of Table 4.

While acknowledging that the sample is small, it appears that wind-fed black holes with supergiant companions are restricted to high spin, $a_{*}>0.8$, in contrast with the broad distribution of spins observed for Roche-lobe-fed black holes with low or intermediate mass companions: four of them have low spins, $a_{*} \approx 0$, two have high spins, $a_{*} \sim 0.7-0.8$, and one has an extreme spin, $a_{*}>0.95$ (see Table 1 in McClintock et al. 2013).

Not only are the persistent black holes all rapidly spinning, they are also relatively massive, $M=11-16 M_{\odot}$ (Table 4 ). By comparison, the masses of the transient black holes are significantly lower, and their mass distribution is remarkably narrow: $7.8 \pm 1.2 M_{\odot}$ (Özel et al. 2010; Farr et al. 2011).

The data in Table 4 highlight a sharp and well-known distinction between the persistent systems and the transient systems, namely, that the secondary stars in the former are far more massive, $M_{2}=20-70 M_{\odot}$ (Table 4); they likewise have much higher temperatures, 30,000-36,000 K (Orosz et al. 2007, $2009,2011)$. The masses and temperatures of the secondaries in the transient systems are typically $<1 M_{\odot}$ and 4000-5000 K; even in exceptional cases, their masses and temperatures are only $M_{2} \lesssim 5 M_{\odot}$ and $T_{\text {eff }, 2} \lesssim 15,000 \mathrm{~K}$ (Charles \& Coe 2006). Finally, we note that for the persistent systems the radii of the secondaries and orbital periods fall in quite narrow ranges (Table 4), while the radii and periods for the transient systems are very broadly distributed, a distinction that is elegantly illustrated in Jerome Orosz's schematic sketch of 21 black hole binaries (see Figure 1 in McClintock et al. 2013).

The persistent black holes were very likely born with their high spins because their host systems are too young for the black holes to have had time to spin up via accretion torques (see Section 7.7 in GOU11 for details). The ages of Cygnus $\mathrm{X}-1$, LMC X-1, and M33 X-7 are $<10$ million years, whereas the spin-up times are $\gtrsim 17$ million years if one assumes the maximum, Eddington-limited accretion rate. Meanwhile, the spin-up times are likely much longer than 17 million years given that the systems are presently radiating at only $\sim 10 \%$ of the Eddington luminosity (Table 4).

The rotational energy of the persistent black holes is enormous, $\sim 2 M_{\odot} c^{2}$ for M33 X-7 and LMC X-1 $>2.8 M_{\odot} c^{2}$ for Cygnus X-1 (Christodoulou \& Ruffini 1971). ${ }^{21}$ Correspondingly, a substantial fraction of the gravitational mass of these 21 By comparison, in its $\sim 10$ billion year lifetime, the energy radiated by the
Sun is $\lesssim 0.001 M_{\odot} c^{2}$. 
black holes is attributable to their rotational energy: $\sim 15 \%$ for M33 X-7 and LMC X-1 and >19\% for Cygnus X-1.

\section{CONCLUSION}

In GOU11, while considering a wide range of systematic effects, including uncertainties in the Novikov-Thorne disk model, we concluded that the spin of the black hole in Cygnus $\mathrm{X}-1$ is extreme: $a_{*}>0.95(3 \sigma)$. Unfortunately, the result was potentially biased by the relatively strong Compton component of emission, the strength of which can be characterized by the fraction $f_{\mathrm{s}}$ of seed photons that are scattered into the power-law component. The three spectra analyzed in GOU11 have $f_{\mathrm{s}}>23 \%$, while $f_{\mathrm{s}} \approx 25 \%$ is the upper limit for reliable application of the continuum-fitting method (Steiner et al. 2009a). Subsequently, Fabian et al. (2012) employed the independent Fe-line method and confirmed that the spin of Cygnus X-1 is $a_{*}>0.95(1 \sigma)$; however, this result is less certain because systematic effects in the model have not been assessed.

Herein, we present a continuum-fitting analysis of six additional spectra, each of which confirms that $a_{*}>0.95(3 \sigma)$. This confirmation is compelling first because sources of systematic error have been thoroughly addressed (see Section 5 herein; Sections 5-7 in GOU11; McClintock et al. 2013). Second, and crucially, five of the spectra, S1-S5, are only moderately Comptonized with scattering fractions $f_{\mathrm{s}}=10 \%-19 \%$, a regime where it has been firmly established that continuumfitting results are reliable. This conclusion is based on studies of two black holes: (1) 33 spectra of H1743-322 with $\bar{f}_{\mathrm{s}}=13.5 \%$ (in the steep power-law state) each gave spins consistent with those obtained for dozens of thermal-state spectra $\left(\bar{f}_{\mathrm{s}}=1 \%-7 \%\right.$; Steiner et al. 2009a); and (2) 25 spectra of XTE J1550-564 with $\bar{f}_{\mathrm{s}}=14.4 \%$ each likewise gave spins consistent with those obtained for dozens of thermal-state spec$\operatorname{tra}\left(\bar{f}_{\mathrm{s}}=2.3 \%\right.$; Steiner et al. 2011). In short, these two studies show that moderately Comptonized spectra with $f_{\mathrm{s}} \sim 15 \%$, like $\mathrm{S} 1-\mathrm{S} 5$, give the same values of spin as spectra that are strongly disk-dominated with $f_{\mathrm{s}} \sim 1 \%-2 \%$.

Our bottom line is that new and more reliable continuum spectra confirm the findings of GOU11 while establishing an even more stringent limit on the extreme spin of Cygnus X-1's black hole: $a_{*}>0.983$ at a confidence level of $3 \sigma(99.7 \%)$.

We thank an anonymous referee for many constructive comments and criticisms, particularly those concerning pileup. We are grateful for allocations of Chandra, RXTE, and Swift observing time granted by Director H. Tananbaum and Project Scientists T. Strohmayer and N. Gehrels, respectively. For help in planning the Chandra observations, we thank M. Nowak and N. Schulz. We also thank M. Nowak, J. Wilms, and Bin-Bin Zhang for discussions on X-ray data analysis, R. Smith for calling the effects of dust scattering to our attention, and S. Yamada for reducing the Suzaku data, and J. G. Xiang for reducing the Chandra TE data. This research has made use of data obtained from the High Energy Astrophysics Science Archive Research Center (HEASARC) at NASA/Goddard Space Flight Center. For technical support in using the Odyssey cluster, L.J.G. thanks the Harvard FAS Sciences Division Research Computing Group. L.J.G. acknowledges the support of NSFC grant (Y211541001, 11333005) and NAOC grant (Y234031001), and is also supported by the Strategic Priority Research Program "The Emergence of Cosmological Structures" of the
Chinese Academy of Sciences, grant No. XDB09000000, J.E.M. acknowledges support from NASA grant NNX11AD08G, J.F.S. has been supported by NASA Hubble Fellowship grant HST-HF-51315.01, and M.H. acknowledges funding from the Bundesministerium für Wirtschaft und Technologie under grant No. DLR 50 OR 0701.

\section{REFERENCES}

Arnaud, K. A. 1996, in ASP Conf. Ser. 101, Astronomical Data Analysis Software and Systems V, ed. G. H. Jacoby \& J. Barnes (San Francisco, CA: ASP), 17

Bardeen, J. M., Press, W. H., \& Teukolsky, S. A. 1972, ApJ, 178, 347

Bolton, C. T. 1972, Natur, 240, 124

Bowyer, S., Byram, E. T., Chubb, T. A., \& Friedman, H. 1965, Sci, 147, 394

Brenneman, L. W., \& Reynolds, C. S. 2006, ApJ, 652, 1028

Canizares, C. R., Davis, J. E., Dewey, D., et al. 2005, PASP, 117, 1144

Charles, P. A., \& Coe, M. J. 2006, in Optical, Ultraviolet and Infrared Observations of X-ray Binaries, ed. W. H. G. Lewin \& M. van der Klis (Cambridge: Cambridge Univ. Press), 215

Christodoulou, D., \& Ruffini, R. 1971, PhRvD, 4, 3552

Dauser, T., Wilms, J., Reynolds, C. S., \& Brenneman, L. W. 2010, MNRAS, 409, 1534

Dickey, J. M., \& Lockman, F. J. 1990, ARA\&A, 28, 215

Duro, R., Dauser, T., Wilms, J., et al. 2011, A\&A, 533, L3

Fabian, A. C., Wilkins, D. R., Miller, J. M., et al. 2012, MNRAS, 424, 217

Farr, W. M., Sravan, N., Cantrell, A., et al. 2011, ApJ, 741, 103

García, J., Dauser, T., Reynolds, C. S., et al. 2013, ApJ, 768, 146

Garmire, G. P., Bautz, M. W., Ford, P. G., Nousek, J. A., \& Ricker, G. R., Jr. 2003, Proc. SPIE, 4851, 28

Gou, L., McClintock, J. E., Liu, J., et al. 2009, ApJ, 701, 1076

Gou, L., McClintock, J. E., Reid, M. J., et al. 2011, ApJ, 742,85

Hanke, M., Wilms, J., Nowak, M. A., Barragán, L., \& Schulz, N. S. 2010, A\&A, 509, L8

Hanke, M., Wilms, J., Nowak, M. A., et al. 2009, ApJ, 690, 330

Kalberla, P. M. W., Burton, W. B., Hartmann, D., et al. 2005, A\&A, 440, 775

Karitskaya, E. A., Agafanov, M. I., Bochkarev, N. G., et al. 2007, A\&AT, 26, 159

King, A. R., Pringle, J. E., \& Livio, M. 2007, MNRAS, 376, 1740

Kulkarni, A. K., Penna, R. F., Shcherbakov, R. V., et al. 2011, MNRAS, 414, 1183

Li, L., Zimmerman, E. R., Narayan, R., \& McClintock, J. E. 2005, ApJS, 157,335

Liu, J., McClintock, J. E., Narayan, R., Davis, S. W., \& Orosz, J. A. 2008, ApJL, 679, L37

McClintock, J. E., Narayan, R., \& Steiner, J. F. 2013, SSRv, arXiv:1303.1583

McClintock, J. E., \& Remillard, R. A. 2006, in Black Hole Binaries, ed. W. H. G. Lewin \& M. van der Klis (Cambridge: Cambridge Univ. Press), 157

McClintock, J. E., Shafee, R., Narayan, R., et al. 2006, ApJ, 652, 518

Mihara, T., Nakajima, M., Sugizaki, M., et al. 2011, PASJ, 63, 623

Miller, J. M., Reynolds, C. S., Fabian, A. C., Miniutti, G., \& Gallo, L. C. 2009, ApJ, 697, 900

Mirabel, I. F., \& Rodrigues, I. 2003, Sci, 300, 1119

Noble, S. C., Krolik, J. H., Schnittman, J. D., \& Hawley, J. F. 2011, ApJ, 743,115

Novikov, I. D., \& Thorne, K. S. 1973, in Black Holes (Les Astres Occlus), ed. C. Dewitt \& B. S. Dewitt (New York: Gordon \& Breach), 343

Orosz, J. A., McClintock, J. E., Aufdenberg, J. P., et al. 2011, ApJ, 742, 84

Orosz, J. A., McClintock, J. E., Narayan, R., et al. 2007, Natur, 449, 872

Orosz, J. A., Steeghs, D., McClintock, J. E., et al. 2009, ApJ, 697, 573

Özel, F., Psaltis, D., Narayan, R., \& McClintock, J. E. 2010, ApJ, 725, 1918

Penna, R. F., McKinney, J. C., Narayan, R., et al. 2010, MNRAS, 408, 752

Predehl, P., \& Schmitt, J. H. M. M. 1995, A\&A, 293, 889

Reid, M. J., McClintock, J. E., Narayan, R., et al. 2011, ApJ, 742, 83

Remillard, R. A., \& McClintock, J. E. 2006, ARA\&A, 44, 49

Reynolds, C. S. 2013, SSRv, arXiv:1302.3260

Romano, P., Campana, S., Chincarini, G., et al. 2006, A\&A, 456, 917

Ross, R. R., \& Fabian, A. C. 2005, MNRAS, 358, 211

Ross, R. R., Fabian, A. C., \& Young, A. J. 1999, MNRAS, 306, 461

Shafee, R., McKinney, J. C., Narayan, R., et al. 2008, ApJL, 687, L25

Smith, R. K., Edgar, R. J., \& Shafer, R. A. 2002, ApJ, 581, 562

Steiner, J. F., McClintock, J. E., \& Reid, M. J. 2012, ApJL, 745, L7 
Steiner, J. F., McClintock, J. E., Remillard, R. A., Narayan, R., \& Gou, L. 2009a, ApJL, 701, L83

Steiner, J. F., McClintock, J. E., Remillard, R. A., et al. 2010, ApJL, 718, L117

Steiner, J. F., Narayan, R., McClintock, J. E., \& Ebisawa, K. 2009b, PASP, 121,1279

Steiner, J. F., Reis, R. C., McClintock, J. E., et al. 2011, MNRAS, 416, 941

Thorne, K. S. 1974, ApJ, 191, 507

Tomsick, J. A., Nowak, M. A., Parker, M., et al. 2014, ApJ, 780, 78
Toor, A., \& Seward, F. D. 1974, AJ, 79, 995

Webster, B. L., \& Murdin, P. 1972, Natur, 235, 37

Weingartner, J. C., \& Draine, B. T. 2001, ApJ, 563, 842

Wilms, J., Allen, A., \& McCray, R. 2000, ApJ, 542, 914

Xiang, J., Lee, J. C., Nowak, M. A., \& Wilms, J. 2011, ApJ, 738, 78

Yamada, S., Torii, S., Mineshige, S., et al. 2013, ApJL, 767, L35

Yamada, S., Uchiyama, H., Dotani, T., et al. 2012, PASJ, 64, 53

Zhang, S. N., Cui, W., \& Chen, W. 1997, ApJL, 482, L155

Zhu, Y., Davis, S. W., Narayan, R., et al. 2012, MNRAS, 424, 2504 